\title{
Hydroxytyrosol protects against myocardial ischemia reperfusion injury by inhibiting mitochondrial permeability transition pore opening
}

\author{
JIAXIN MIAO ${ }^{1}$, ZIJUN HUANG ${ }^{1}$, SHUANG LIU ${ }^{1}$, XUYING LI $^{1}$, PENGYU JIA ${ }^{1}$, \\ YUXUAN GUO $^{1}$, NAN WU ${ }^{2}$ and DALIN JIA ${ }^{1}$ \\ ${ }^{1}$ Department of Cardiology and ${ }^{2}$ Central Laboratory, The First Affiliated Hospital of China Medical University, \\ Shenyang, Liaoning 110001, P.R. China
}

Received June 1, 2018; Accepted October 19, 2018

DOI: $10.3892 / \mathrm{etm} .2018 .7016$

\begin{abstract}
Hydroxytyrosol (HT), a phenolic compound extracted from olive oil, is reported to protect against myocardial ischemia reperfusion injury (MIRI), but its mechanism has not been fully elucidated. The mitochondria permeability transition pore (MPTP) is an important therapeutic target for MIRI. The present study aimed to investigate the role of MPTP in the cardioprotection of HT. Isolated rat hearts were mounted on a Langendorff apparatus and subjected to $30 \mathrm{~min}$ of ischemia followed by 120 min of reperfusion to mimic a MIRI model. Isolated hearts were pretreated with different doses of HT $(10,100$ and $1,000 \mu \mathrm{M})$ for $10 \mathrm{~min}$ prior to ischemia. Myocardial infarct size was detected using TTC staining. Changes in myocardial cell structure were observed using hematoxylin and eosin staining. MPTP opening was detected spectrophotometrically. Myocardial cell apoptosis was observed with terminal deoxynucleotidyl-transferase-mediated dUTP nick end labeling assays. The expression of apoptosis-associated proteins was measured by western blot analysis. The data revealed that HT $(100$ and $1,000 \mu \mathrm{M})$ treatment significantly alleviated pathological damage in ischemic myocardium and reduced myocardial infarct size compared with the untreated control. However, no significant difference was observed in the $10 \mu \mathrm{M}$ HT treatment group compared with the untreated control. It was further revealed that HT decreased the B cell lymphoma-2 (Bcl-2)-like protein $4(\mathrm{Bax}) / \mathrm{Bcl}-2$ ratio, suppressed MPTP opening and
\end{abstract}

Correspondence to: Dr Dalin Jia, Department of Cardiology, The First Affiliated Hospital of China Medical University, 155 North Nanjing Street, Shenyang, Liaoning 110001, P.R. China E-mail: jd12001@126.com

Dr Nan Wu, The Central Laboratory, The First Affiliated Hospital of China Medical University, 155 North Nanjing Street, Shenyang, Liaoning 110001, P.R. China

E-mail: imwunan@163.com

Key words: myocardial ischemia reperfusion injury, hydroxytyrosol, mitochondrial permeability transition pore, apoptosis subsequently decreased the expression of cytochrome $c$, cleaved caspase- -9 and -3 , thereby inhibiting apoptosis. Additionally, the beneficial effects of HT on MIRI were reversed by atractyloside, which induces MPTP opening. In conclusion, the present study demonstrated that HT inhibited MPTP opening, partially via modulation of $\mathrm{Bax}$ and $\mathrm{Bcl}-2$, thereby protecting against MIRI and thereby providing a pharmacological basis for future research and treatment of MIRI.

\section{Introduction}

Acute myocardial infarction (AMI) is the leading cause of morbidity and mortality worldwide (1). Reperfusion, the restoration of blood flow, has been considered the most effective treatment of ischemic heart disease, in particular AMI (2). However, reperfusion has further been referred to as a double-edged sword, as reperfusion may cause aggravation of myocardial injury, termed myocardial ischemia reperfusion injury (MIRI) (3). With the wide application of reperfusion therapy, including drug thrombolysis, percutaneous coronary intervention and coronary artery bypass grafting, the elucidation of the mechanism of MIRI prevention has become imperative (4-7).

The mitochondrial permeability transition pore (MPTP) is a non-specific channel located in the inner mitochondrial membrane (8). MPTP remains closed during ischemia, but rapidly opens following the commencement of reperfusion (9). MPTP opening is considered an important mechanism of MIRI (10). Additionally, the inhibition of MPTP opening by cyclosporine A attenuates MIRI (11). Therefore, MPTP is considered an important therapeutic target for the prevention of MIRI.

Hydroxytyrosol (HT), known as 3,4-dihydroxyphenyl ethanol, is a phenolic compound extracted from Mediterranean virgin olive oil (12). Biological effects of HT include the suppression of oxidative stress, inflammation and tumor formation, and protection of the cardiovascular system and neurological function (13-17). HT serves a role in the protection against liver ischemia/reperfusion injury in mice $(18,19)$. Pei et al (20) revealed that HT affects MIRI via the phosphatidylinositol-4,5-bisphosphate 3 kinase (PI3K)/protein kinase B 
(Akt) signaling pathway. However, the effect of HT on MPTP in MIRI remains unknown. Therefore, the present study suggested that HT attenuated MIRI by inhibiting MPTP opening.

The aim of the present study was to investigate the effects of HT on MIRI in an isolated rat heart model and to further explore the role of MPTP in the cardioprotection of HT.

\section{Materials and methods}

Experimental animals and reagents. A total of 100 healthy male Wistar rats (age, 4 weeks; weight, $250 \pm 20 \mathrm{~g}$ ) were purchased from Changsheng Biotechnology Co., Ltd. (Beijing, China). Rats were housed in environmentally controlled conditions (20-25 ${ }^{\circ} \mathrm{C}, 5-65 \%$ relative humidity, with a $12-\mathrm{h}$ light/dark cycle) with a common 1 week acclimatization period. All rats had access to fresh food and water ad libitum. The procedures for handling and caring for animals adhered to the guidelines in compliance with the Guide for the Care and Use of Laboratory Animals (21). The experimental protocol was approved by the Institutional Ethics Committee of China Medical University (Shenyang, China).

HT was purchased from Dalian Meilun Biology Technology Co., Ltd. (Dalian, China). Atractyloside (ATR) and 2,3,5-triphenyltetrazolium chloride (TTC) were purchased from Sigma-Aldrich (Merck KGaA, Darmstadt, Germany).

Establishing MIRI in isolated rat hearts. Pentobarbital sodium $(100 \mathrm{mg} / \mathrm{kg})$ was administered intravenously to anesthetize the rats. Heparin $(1,500 \mathrm{IU} / \mathrm{kg})$ was injected intravenously to prevent intracoronary clot formation. Following opening of the thoracic cavity, hearts were swiftly removed and immediately immersed in ice-cooled heparinized Krebs-Henseleit $(\mathrm{K}-\mathrm{H})$ buffer $\left(0.15 \mathrm{~mol} / \mathrm{l} \mathrm{NaCl}, 0.006 \mathrm{~mol} / \mathrm{l} \mathrm{KCl}, 0.002 \mathrm{~mol} / 1 \mathrm{CaCl}_{2}\right.$, $0.002 \mathrm{~mol} / 1 \mathrm{NaHCO}_{3}$ ) and saturated with $100 \%$ oxygen (22). Isolated hearts were transferred to a Langendorff perfusion system (Taimeng Science and Technology Ltd., Chengdu, China) to perform heart perfusion with K-H solution, saturated with $95 \% \mathrm{O}_{2}+5 \% \mathrm{CO}_{2}$ at $37^{\circ} \mathrm{C}$. A water-filled latex balloon was inserted into the left ventricle through the left atrium and connected to a pressure transducer for pressure measurement. All isolated hearts were continually perfused with K-H solution for $10 \mathrm{~min}$ stabilization, prior to the commencement of ischemia. All isolated rat hearts were subjected to $30 \mathrm{~min}$ global ischemia, followed by $120 \mathrm{~min}$ of reperfusion to generate the MIRI model.

Experimental protocol. The experimental protocol consisted of two phases. In the first phase, 40 rats were divided into the following 4 groups with 10/group: i) Ischemia reperfusion group (IR): As described above; ii) $10 \mu \mathrm{M}$ HT treatment group (HT $10 \mu \mathrm{M})$ : Isolated hearts were perfused with $10 \mu \mathrm{M}$ HT for 10 min and K-H solution for 5 min prior to induction of ischemia; iii) $100 \mu \mathrm{M}$ HT treatment group (HT $100 \mu \mathrm{M}$ ): Isolated hearts were perfused with $100 \mu \mathrm{M}$ HT for $10 \mathrm{~min}$ and K-H solution for $5 \mathrm{~min}$ prior to induction of ischemia; iv) $1,000 \mu \mathrm{M}$ HT treatment group (HT 1,000 $\mu \mathrm{M}$ ): Isolated hearts were perfused with $1,000 \mu \mathrm{M}$ HT for $10 \mathrm{~min}$ and $\mathrm{K}-\mathrm{H}$ solution for $5 \mathrm{~min}$ prior to induction of ischemia.

According to the results of the first phase, $100 \mu \mathrm{M}$ HT was chosen for the second phase. A total of 60 rats were divided into the following 3 groups with 20/group: i) IR group; ii) HT
$100 \mu \mathrm{M}$ group: The heart rate was stabilized and the heart was perfused with HT for $10 \mathrm{~min}$, then with K-H solution for $5 \mathrm{~min}$, ischemia/reperfusion was performed as in IR group. iii) $100 \mu \mathrm{M}$ HT in combination with ATR treatment group (HT $100 \mu \mathrm{M}+\mathrm{ATR}$ ): $5 \mathrm{mg} / \mathrm{kg}$ ATR was injected intraperitoneally 30 min prior to extraction of the heart, the remainder of the procedure was as described for the HT $100 \mu \mathrm{M}$ group.

Cardiac function monitoring. Change in cardiac function was evaluated monitoring heart rate (HR) and coronary flow (CF). $\mathrm{HR}$ and $\mathrm{CF}$ were measured prior to ischemia, and 30 and $60 \mathrm{~min}$ post initiation of reperfusion.

Hematoxylin and eosin (HE) staining. Following reperfusion, hearts were harvested. Heart tissues were fixed in $4 \%$ paraformaldehyde for 24-72 $\mathrm{h}$ at room temperature and washed with flowing water for $4 \mathrm{~h}$. Heart tissue samples were subsequently dehydrated in an ascending ethanol series (70\% ethanol for $2 \mathrm{~h}$, $80 \%$ ethanol overnight, $90 \%$ ethanol for 2 h, $100 \%$ ethanol I for $1 \mathrm{~h}$ and $100 \%$ ethanol II for $1 \mathrm{~h}$ ) at room temperature. The tissue samples were embedded in paraffin embedded and the paraffin-embedded tissue samples were cut into 5- $\mu \mathrm{m}$-thick sections. The tissue sections were subsequently deparaffinized in xylene I for $15 \mathrm{~min}$ and xylene II for $15 \mathrm{~min}$ at room temperature and rehydrated in a descending ethanol series $(100 \%$ ethanol I for $5 \mathrm{~min}, 100 \%$ ethanol II for $5 \mathrm{~min}, 95 \%$ ethanol for $2 \mathrm{~min}, 85 \%$ ethanol for $2 \mathrm{~min}, 75 \%$ ethanol for $2 \mathrm{~min}$ ) and distilled water for $2 \mathrm{~min}$ at room temperature. Deparaffinized section were incubated with hematoxylin solution for $5 \mathrm{~min}, 1 \%$ hydrochloric acid alcohol for $3 \mathrm{sec}$ and eosin solution for $3 \mathrm{~min}$ at room temperature. Pathological changes were observed under a light microscope (Olympus BX51; Olympus Corporation, Tokyo, Japan; magnification, x400).

Measurement of myocardial infarct size. Following reperfusion, the hearts were removed, frozen at $-20^{\circ} \mathrm{C}$ for $1 \mathrm{~h}$ and sliced into 1-2 $\mathrm{mm}$ thick sections. The sections were incubated in a $1 \%$ TTC solution for $20 \mathrm{~min}$ at $37^{\circ} \mathrm{C}$. Tissue sections were washed with 1X PBS and fixed in 4\% paraformaldehyde overnight at room temperature. Images of the stained slices were captured using a digital camera and analyzed using Image J2X analysis software (National Institutes of Health, Bethesda, MD, USA). The severity of the myocardial infarction was indicated by the ratio of the infarct area to the total area.

Apoptosis. Myocardial apoptosis was detected by terminal deoxynucleotidyl-transferase-mediated dUTP nick end labeling (TUNEL) assay using the In Situ Cell Death Detection kit (cat. no. 11684817910; Roche Diagnostics, Indianapolis, IN, USA), as previously described (23). Apoptotic cells were observed under a light microscope (magnification, $\mathrm{x} 400$ ) in three randomly selected fields. Image-Pro Plus (version 6.0; Media Cybernetics, Inc., Rockville, MD, USA) was used for cell counting.

MPTP sensitivity to $\mathrm{Ca}^{2+}$. Mitochondria were isolated from heart tissues using the Tissue Mitochondria Isolation kit (Beyotime Institute of Biotechnology, Shanghai, China), according to the manufacturer's protocol. The sensitivity of MPTP to $\mathrm{Ca}^{2+}$ was determined using the Purified 

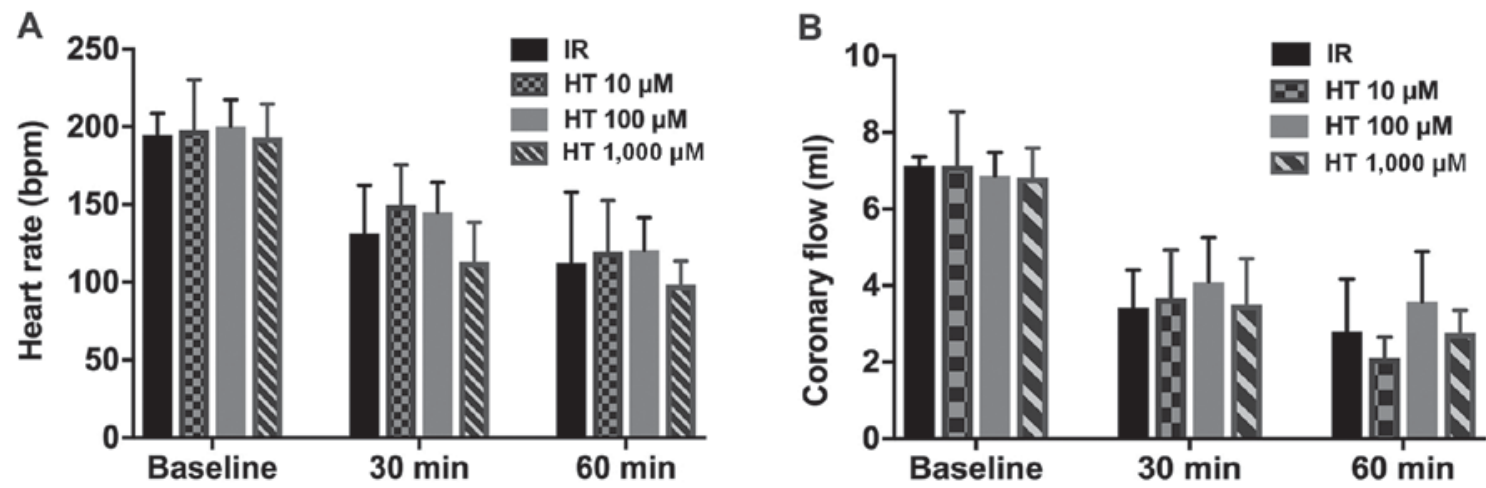

Figure 1. HT treatment does not alter HR and CF. (A) HR and (B) CF determined in animals treated with HT at $0,10,100$ and 1,000 $\mu \mathrm{M}$ at various times following IR ( $n=6 /$ group). IR, ischemia reperfusion; HT, hydroxytyrosol; HR, heart rate; $\mathrm{CF}$, coronary flow.

Mitochondrial Membrane Pore Channel Colorimetric Assay kit (cat. no. GMS10095; Shanghai Genmed Pharmaceutical Technology Co., Ltd., Shanghai, China), according to the manufacturer's protocol. The larger the $\min / \mathrm{max}$ ratio, the lower the sensitivity of MPTP opening to $\mathrm{Ca}^{2+}$, conversely, the smaller the $\mathrm{min} / \mathrm{max}$ ratio, the higher the sensitivity of MPTP opening to $\mathrm{Ca}^{2+}$.

Western blot analysis. The left ventricle tissues were homogenized with radioimmunoprecipitation buffer (Beyotime Institute of Biotechnology) and protease inhibitor phenylmethanesulfonyl fluoride (Beyotime Institute of Biotechnology) on ice for $20 \mathrm{~min}$. Proteins were extracted from lysates following centrifugation at $13,000 \times \mathrm{g}$ for $15 \mathrm{~min}$ at $4^{\circ} \mathrm{C}$. Total protein was quantified using the Enhanced BCA Protein Assay kit (Beyotime Institute of Biotechnology). Subsequently, $50 \mu \mathrm{g}$ protein was denatured at $100^{\circ} \mathrm{C}$ for $10 \mathrm{~min}$, and separated via SDS-PAGE on a $10 \%$ gel. The separated proteins were transferred onto polyvinylidene difluoride membranes and blocked with $5 \%$ skimmed milk for $1 \mathrm{~h}$ at room temperature. The membranes were incubated with primary antibodies against B-cell lymphoma 2 (Bcl-2)-associated X protein (Bax; 1:1,000; cat. no. WL01637), Bcl-2 (1:1,000; cat. no. WL01556; both Shenyang Wan Biotechnology Co., Ltd., Shenyang, China), cytochrome $c(1: 1,000$; cat. no. ab90529), apoptotic protease activating factor-1 (APAF-1; 1:1,000; cat. no. ab2001), cleaved caspase-3 (1:1,000; cat. no. ab2302; all Abcam, Cambridge, UK), cleaved caspase-9 (1:1,000; cat. no. 40503-1; Signalway Anitbody LLC, College Park, MD, USA) and $\beta$-actin (1:1,000; cat. no. TA-09; OriGene Technologies, Inc., Beijing, China) overnight at $4^{\circ} \mathrm{C}$. Following primary incubation, the membranes were incubated with horseradish peroxidase-labeled goat anti-rabbit immunoglobulin G (1:4,000; cat. no. E030120-01) or goat anti-mouse immunoglobulin G (1:4,000; cat. no. E030110-01; both EarthOx Life Sciences, Millbrae, CA, USA) at $37^{\circ} \mathrm{C}$ for $2 \mathrm{~h}$. Protein bands were visualized using BeyoECL Star (Beyotime Institute of Biotechnology), according to the manufacturer's protocol. Levels of phosphorylated proteins were normalized to the corresponding total protein levels. Relative densitometry was calculated using ImageJ (version 1.50e; National Institutes of Health).

Statistical analysis. Data are expressed as the mean \pm standard deviation from three independent experiments. All statistical analysis was performed using SPSS 17.0 (SPSS, Inc., Chicago, IL, USA). Differences between groups were evaluated using one-way analysis of variance followed by Fisher's least significant difference tests. $\mathrm{P}<0.05$ was considered to indicate a statistically significant difference.

\section{Results}

Effects of HT on HR and CF. As presented in Fig. 1, no statistical significance was detected for $\mathrm{HR}$ or $\mathrm{CF}$ in rats of the various experimental groups at various times $(\mathrm{P}>0.05)$.

Effects of HT on myocardial injury. HE staining revealed that HT (100 and 1,000 $\mu \mathrm{M})$ pretreatment markedly alleviated pathological damage in the ischemic myocardium; however, pretreatment with $10 \mu \mathrm{M}$ HT did not reveal any alleviation of pathological damage in the ischemic myocardium (Fig. 2A). Results of the TTC assay revealed the infarct size, which is presented as white tissue sections compared with red normal tissue sections (Fig. 2B). The myocardial infarct size of the 100 and 1,000 $\mu \mathrm{M}$ HT groups was significantly decreased compared with the IR group $(30.9 \pm 2.9$ and $32.3 \pm 3.2$ vs. $54.3 \pm 3.0 \% ; \mathrm{P}<0.05$; Fig. 2C). However, pretreatment with $10 \mu \mathrm{M}$ HT revealed no significant change in the myocardial infarct size compared with the IR group ( $P>0.05$; Fig. $2 \mathrm{C}$ ). The protective effect on MIRI was not improved when increasing HT from 100 to $1,000 \mu \mathrm{M}$. Therefore, $100 \mu \mathrm{M}$ HT was selected for second-stage experiments.

ATR reverses protective effects of $H T$. To elucidate the role of $\mathrm{HT}$ in cardioprotection during MIRI, the effects of ATR, a MPTP opener, were investigated. The results revealed that myocardial injury was worsened in the HT $100 \mu \mathrm{M}+$ ATR group compared with the HT $100 \mu \mathrm{M}$ group (Fig. 3A). Myocardial infarct size was significantly increased in the HT $100 \mu \mathrm{M}+$ ATR group compared with the HT $100 \mu \mathrm{M}$ group $(52.8 \pm 7.8$ vs. $30.9 \pm 2.9 \%$; $\mathrm{P}<0.05$; Fig. 3B and C).

Effects of HT on MPTP opening. MPTP opening is generally induced by $\mathrm{Ca}^{2+}$ and the sensitivity of MPTP to $\mathrm{Ca}^{2+}$ is an indicator for MPTP opening (24-26). The results indicated that changes in $\mathrm{Ca}^{2+}$ induced MPTP opening. Isolated mitochondria from the HT $100 \mu \mathrm{M}$ group were significantly more resistant to 
A

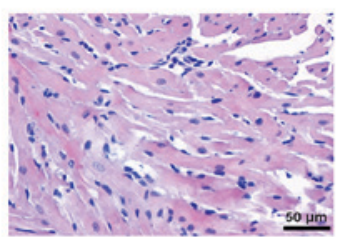

IR

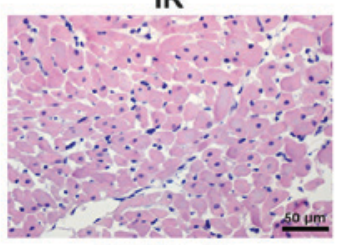

HT $10 \mu \mathrm{M}$

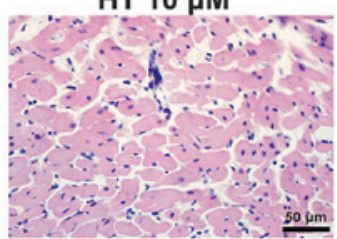

HT $100 \mu \mathrm{M}$

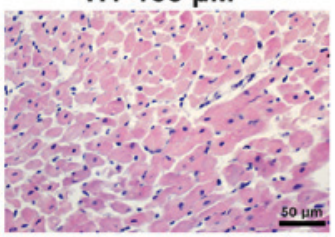

HT $1000 \mu \mathrm{M}$
B

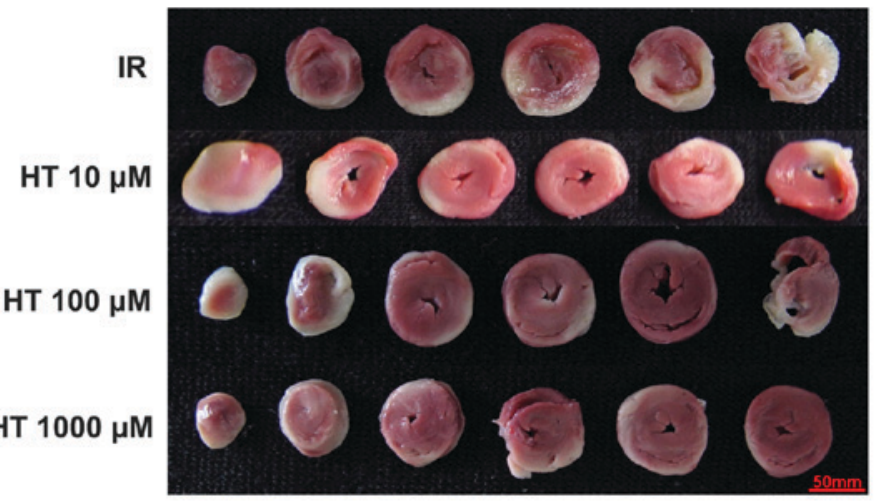

C

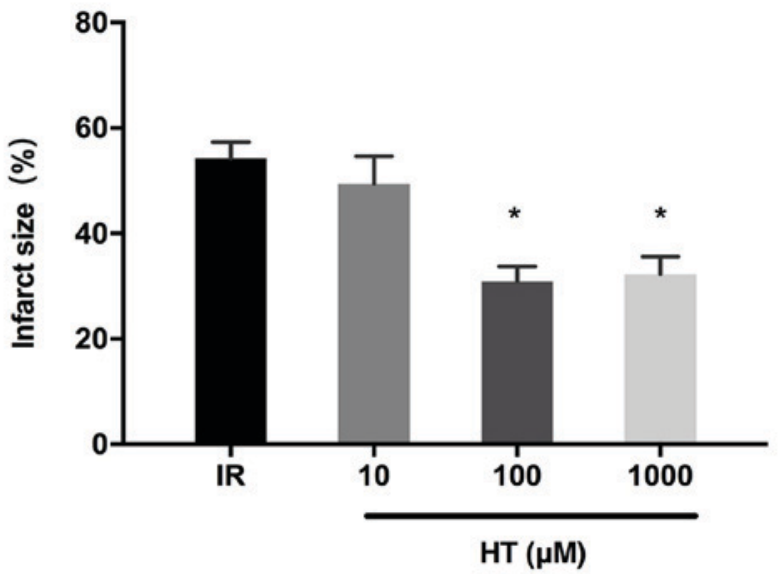

Figure 2. Effects of HT on myocardial ischemia reperfusion injury. (A) Pathological changes of the ischemic myocardium stained by hematoxylin-eosin (magnification, $x 400$; scale bar, $50 \mu \mathrm{m}$ ). (B) Heart tissue slices stained with 2,3,5-triphenyltetrazolium chloride visualizing the size of the myocardial infarct (scale bar, $50 \mathrm{~mm}$ ). (C) Statistical analysis of the myocardial infarct size $(\mathrm{n}=6)$. ${ }^{*} \mathrm{P}<0.05$ vs. IR. IR, ischemia reperfusion; HT, hydroxytyrosol.

A

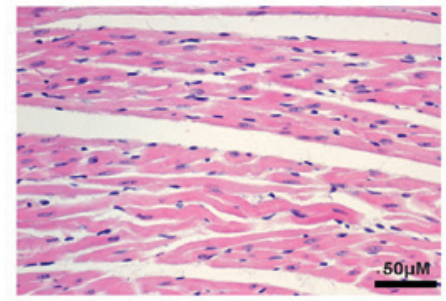

IR

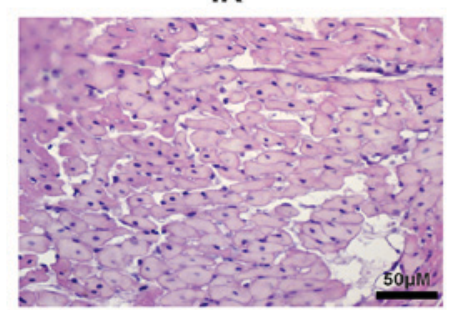

HT $100 \mu \mathrm{M}$

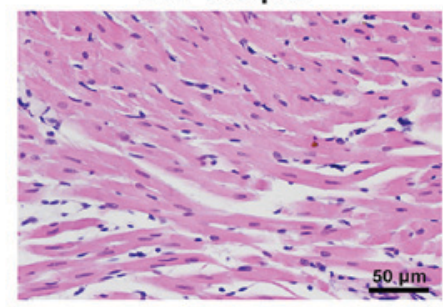

HT $100 \mu \mathrm{M}+\mathrm{ATR}$
B

HT $100 \mu \mathrm{M}+\mathrm{ATR}$

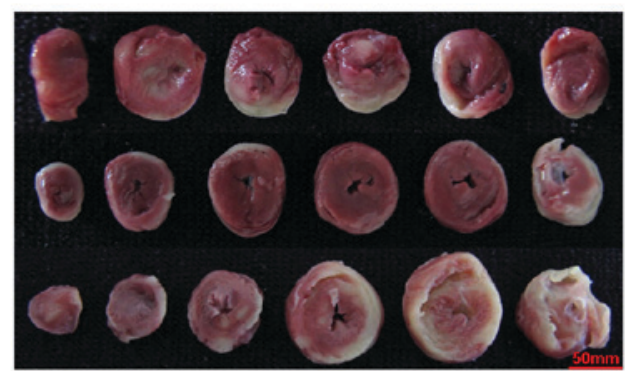

C

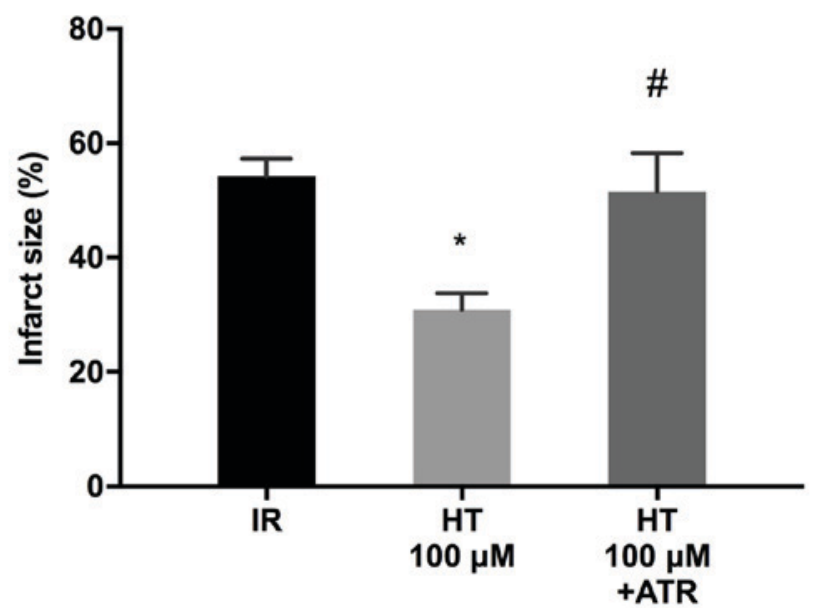

Figure 3. Effects of ATR on HT cardioprotection in myocardial ischemia reperfusion injury. (A) Pathological changes of the ischemic myocardium stained with hematoxylin-eosin (magnification, $\mathrm{x} 400$; scale bar, $50 \mu \mathrm{m}$ ). (B) Heart tissue slices stained with 2,3,5-triphenyltetrazolium chloride visualizing the size of the myocardial infarct (scale bar, $50 \mathrm{~mm}$ ). (C) Statistical analysis of the myocardial infarct sizes ( $\mathrm{n}=6$ ). ${ }^{*} \mathrm{P}<0.05 \mathrm{vs}$. IR and ${ }^{\#} \mathrm{P}<0.05 \mathrm{vs}$. $100 \mu \mathrm{M}$ HT. IR, ischemia reperfusion; HT, hydroxytyrosol; ATR, atractyloside. 
A

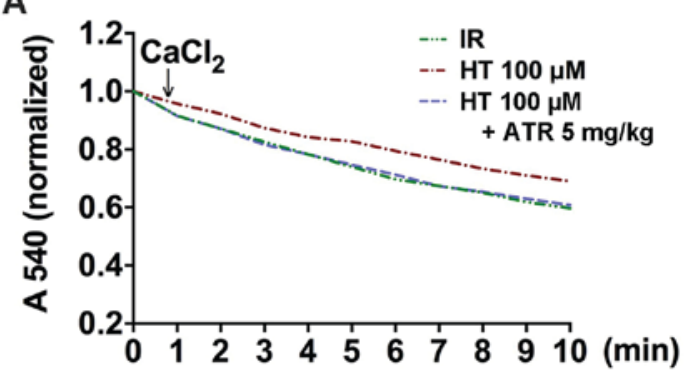

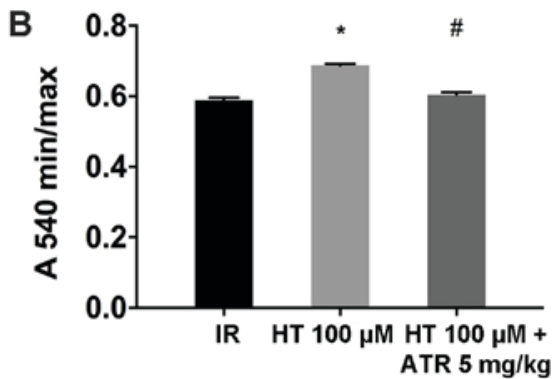

Figure 4. Effects of HT on MPTP opening. $\mathrm{Ca}^{2+}$ sensitivity of MPTP was determined using purified mitochondrial membrane pore channel colorimetric assay. (A) Sensitivity of MPTP opening to $\mathrm{Ca}^{2+}$. (B) Alterations in MPTP opening in response to $\mathrm{Ca}^{2+}(\mathrm{n}=6)$. ${ }^{*} \mathrm{P}<0.05$ vs. IR and ${ }^{*} \mathrm{P}<0.05$ vs. $100 \mu \mathrm{M}$ HT. IR, ischemia reperfusion; HT, hydroxytyrosol; ATR, atractyloside.

A

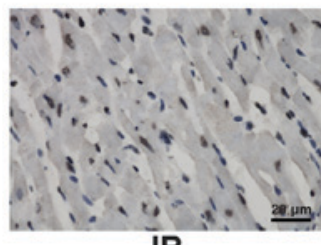

IR

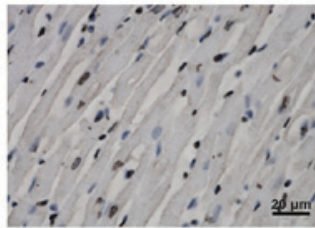

HT $100 \mu \mathrm{M}+\mathrm{ATR}$

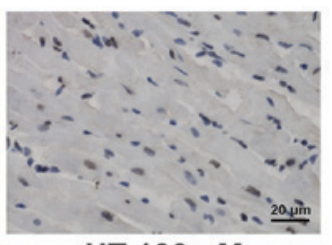

HT $100 \mu \mathrm{M}$
B

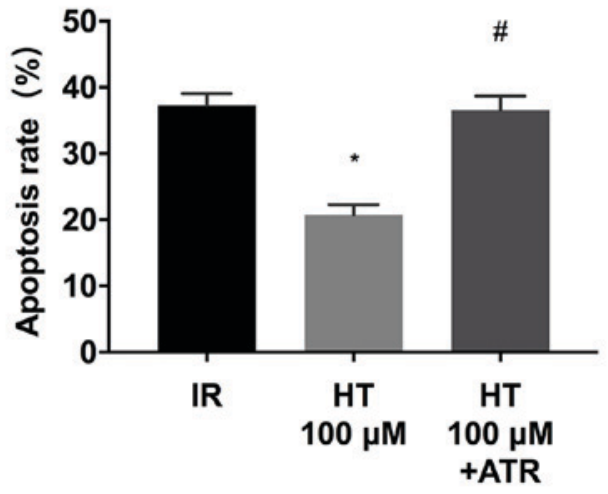

Figure 5. Effects of HT on apoptosis. (A) Apoptosis was detected by terminal deoxynucleotidyl-transferase-mediated dUTP nick end labeling assay (magnification, $\mathrm{x} 400$; scale bar, $20 \mu \mathrm{m})$. (B) Statistical analysis of apoptosis rates $(\mathrm{n}=6)$. ${ }^{*} \mathrm{P}<0.05$ vs. IR and ${ }^{\#} \mathrm{P}<0.05$ vs. $100 \mu \mathrm{M}$ HT. IR, ischemia reperfusion; HT, hydroxytyrosol; ATR, atractyloside.

stimulation by $\mathrm{Ca}^{2+}$ compared with the IR group, suggesting that HT inhibited MPTP opening (Fig. 4A and B). Additionally, the resistance of the isolated mitochondria to $\mathrm{Ca}^{2+}$ was decreased in the HT $100 \mu \mathrm{M}+\mathrm{ATR}$ group compared with the HT $100 \mu \mathrm{M}$ group (Fig. 4B).

Effects of HT on the mitochondrial apoptotic pathway. MPTP opening leads to the release of cytochrome $c$ and activation of caspase-9 and -3 , which results in apoptosis (27). Results from the TUNEL assay revealed that $100 \mu \mathrm{M}$ HT significantly decreased the rate of apoptosis ( $\mathrm{P}<0.05 ;$ Fig. $5 \mathrm{~A}$ and $\mathrm{B})$. In addition, compared with HT $100 \mu \mathrm{M}$ group, the apoptosis rate in the HT $100 \mu \mathrm{M}+$ ATR group increased by $15.8 \%$ (Fig. 5). Similarly, western blot analysis revealed that $100 \mu \mathrm{M}$ HT significantly decreased cytochrome $c$, cleaved caspase- 9 and -3 protein levels compared with the IR group $(\mathrm{P}<0.05 ;$ Fig. 6$)$. In addition, the cytochrome $c$, cleaved caspase- 9 and -3 levels in the HT $100 \mu \mathrm{M}+$ ATR group were significantly increased compared with the $100 \mu \mathrm{M}$ HT group (Fig. 6). The rate of apoptosis and expression level of apoptosis-associated proteins was not significantly different in the ATR-treated group compared with the IR group, suggesting that ATR reversed cardioprotective effects exerted by HT.

Effects of HT on Bax and Bcl-2 protein expression. The $\mathrm{Bcl}-2$ protein family is a key regulator in MPTP opening (28).
Western blot analysis demonstrated that treatment with $100 \mu \mathrm{M}$ HT significantly decreased Bax expression and Bax/Bcl-2 compared with the IR group $(\mathrm{P}<0.05$; Fig. 7). Additionally, $\mathrm{Bcl}-2$ expression was significantly increased compared with the IR group $(\mathrm{P}<0.05$; Fig. 7).

\section{Discussion}

In the present study, treatment with the pharmacological agent HT at 100 or $1,000 \mu \mathrm{M}$ was revealed to reduce the myocardial infarction area and damage to the myocardium in rats compared with the untreated animals, which suggested that HT may protect against MIRI. However, there was no effect for $10 \mu \mathrm{M}$ HT. Additionally, there was no significant difference in the cardioprotection exerted by 100 and 1,000 $\mu \mathrm{M}$ HT. Therefore, it was suggested that a dose-associated effect of HT occurred at lower doses $(10-100 \mu \mathrm{M})$, which is consistent with a previous study by Pan et al (18). Pei et al (20) used SD rats to perform in vivo cardiac ischemia for 30 min followed by reperfusion for $3 \mathrm{~h}$. In their study, rats were intraperitoneally injected with HT at a concentration of $20 \mathrm{mg} / \mathrm{kg}$ during ischemia. The results revealed that HT attenuated MIRI via the PI3K/Akt signaling pathway, and protected functional parameters of the heart. However, in the present study, no difference in HR or CF of rats from various groups was observed at various times. Reasons for this disparity may include differences between in vivo and 
A

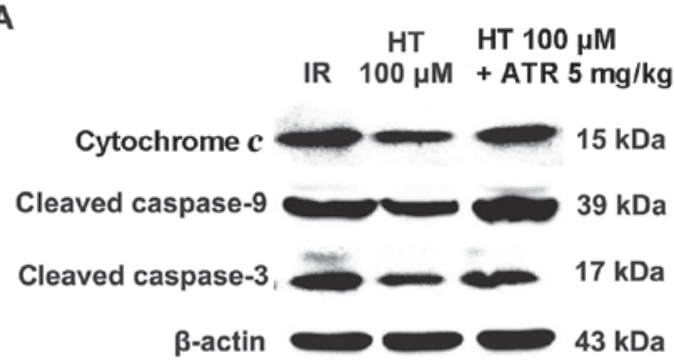

C

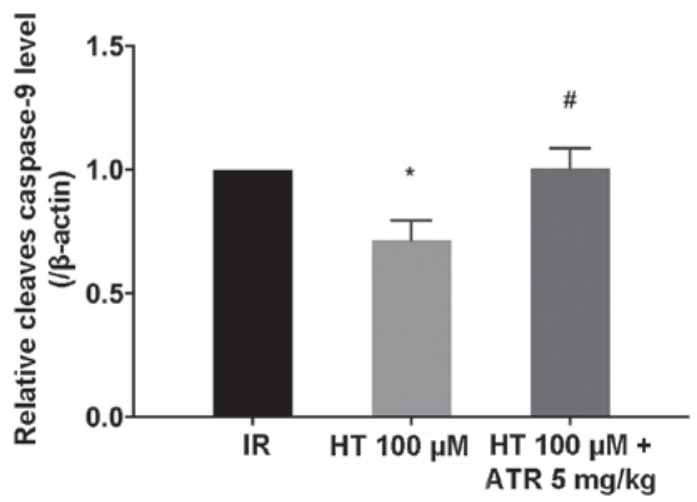

B
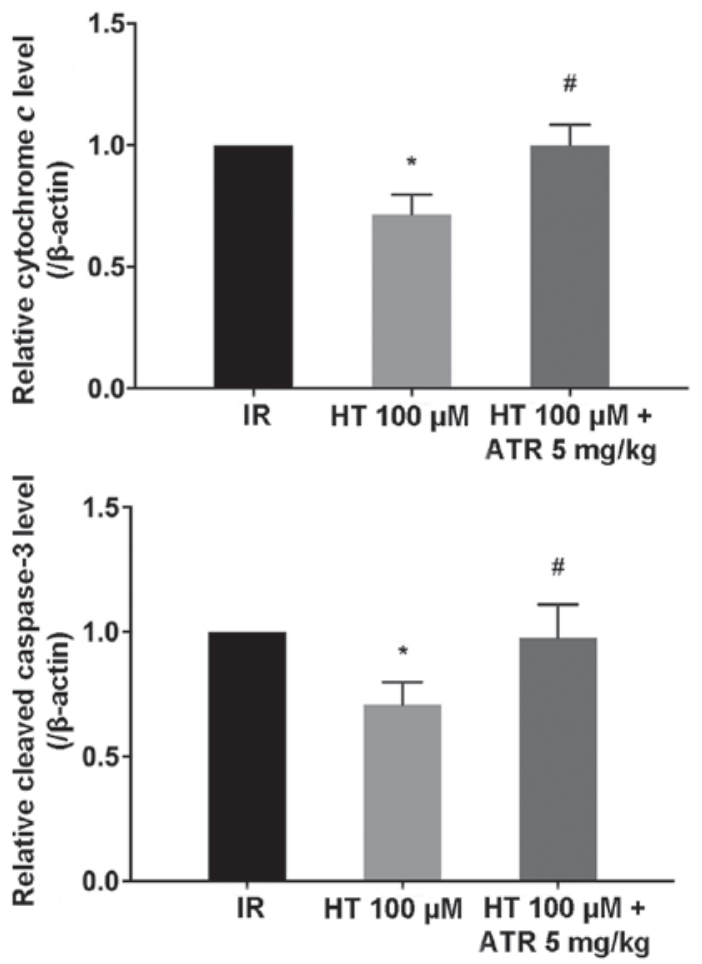

Figure 6. Effects of HT on the mitochondrial apoptotic pathway. (A) Cytochrome $c$, cleaved caspase-9 and -3 levels detected by western blotting. Statistical analysis of the relative expression of (B) cytochrome $c$, (C) cleaved caspase-9 and (D) cleaved caspase-3 (n=6). ${ }^{*} \mathrm{P}<0.05$ vs. IR and ${ }^{\#} \mathrm{P}<0.05$ vs. $100 \mu \mathrm{M}$ HT. IR, ischemia reperfusion; HT, hydroxytyrosol; ATR, atractyloside.

A

Bax

IR

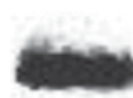

HT $100 \mu \mathrm{M}$

Bcl-2

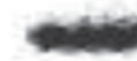

$21 \mathrm{kDa}$

$\beta$-actin
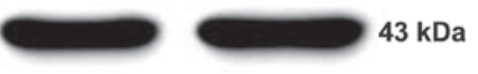

C

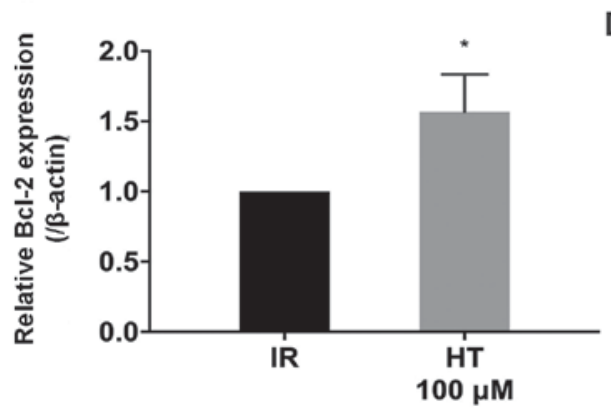

B

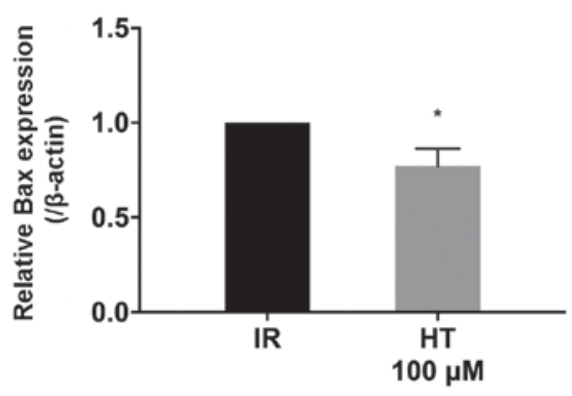

D

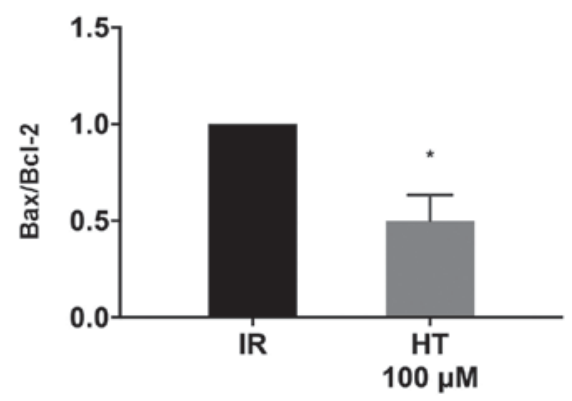

Figure 7. Effects of $\mathrm{HT}$ on the Bcl-2 protein family. (A) Bax and Bcl-2 levels detected by western blotting. Statistical analysis of the relative expression of (B) Bax, (C) Bcl-2 and (D) Bax/Bcl-2 (n=6). "P<0.05 vs. IR. IR, ischemia reperfusion; HT, hydroxytyrosol; Bcl-2, B cell lymphoma-2; Bax, Bcl-2-like protein 4.

in vitro models, differences in rat species, duration of procedures and dosing methods.

To the best of our knowledge, this is the first study to demonstrate the effect of HT on the inhibition of MPTP in MIRI. The opening of MPTP causes irreversible damage to the heart (29). According to previous studies, core components of MPTP are voltage-dependent anion channels (VDAC), adenine nucleotide transporter and cyclophilin-D $(30,31)$. The opening of MPTP is induced by insufficient intracellular adenosine triphosphate synthesis, reactive oxygen species-induced oxidative stress, and $\mathrm{Ca}^{2+}$ and phosphate accumulation $(32,33)$. Several studies have revealed that MIRI is closely associated to MPTP opening (34-36). Pretreatment with several pharmacological agents, including irisin, melatonin and carnosic 
acid, have been demonstrated to alleviate MIRI via inhibition of MPTP opening (37-39). In the present study, it was demonstrated that HT inhibited MPTP opening during MIRI. $\mathrm{Ca}^{2+}$ treatment induces MPTP opening (40); compared with the IR group, it was revealed that isolated mitochondria from rat hearts pretreated with HT had a higher resistance to $\mathrm{Ca}^{2+}$ stimulation, which indicated that HT inhibited MPTP opening. Additionally, it was revealed that HT pretreatment reduced cytochrome $c$, cleaved caspase- 9 and -3 levels and decreased the rate of apoptosis. These observations were similar to results reported by Soni et al (41) studying rat brains. All protective effects of HT were abolished with ATR treatment, which strongly suggested that HT protected against MIRI by inhibiting MPTP opening.

The Bcl-2 protein family is an important constituent of the apoptotic pathway $(42,43)$ and serves an important regulatory role in MPTP opening (44). Members of the Bcl-2 family include the anti-apoptotic protein $\mathrm{Bcl}-2$ and the pro-apoptotic protein $\mathrm{Bax}(45,46)$. Interactions of $\mathrm{Bcl}-2$ and $\mathrm{Bax}$ with VDAC regulate MPTP opening; Bax facilitates MPTP opening by binding to VDAC, while Bcl-2 inhibits binding of Bax to VDAC (28). Bcl-2 and Bax co-express in tissue cells and MPTP opening is closely associated with the ratio of Bax to Bcl-2 (47). In the present study, it was revealed that HT pretreatment enhanced Bcl-2 expression in MIRI and decreased Bax expression and Bax/Bcl-2 levels. This demonstrated that HT inhibited MPTP opening by regulating $\mathrm{Bcl}-2$ and Bax expression, which is consistent with a previous study by Liu and Dong (39). Notably, a recent study suggested that phosphorylated-Akt inhibits MPTP opening by regulating the Bcl-2 protein family (48). Furthermore, Pei et al (20) demonstrated that HT protects the rat myocardium from MIRI via direct activation of the PI3K/Akt signaling pathway. It was therefore further suggested that the PI3K/Akt/Bcl-2 signaling pathway may serve an important role in the inhibition of MPTP opening by HT. This hypothesis requires to be investigated in further studies for confirmation.

There are limitations to the present study. The isolated rat heart model used was deprived of neural and humoral regulation and may not completely mimic pathophysiological changes that occur during MIRI and in vivo cardioprotective effects of HT require further validation. The present study solely demonstrated that HT inhibited MPTP opening via Bcl-2; upstream targets of the MPTP pathway, including PI3K/Akt, glycogen synthase kinase $3 \beta$ and Janus kinase/signal transducer and activator of transcription pathways require further investigation.

In conclusion, the present study demonstrated for the first time that HT protected against MIRI by inhibiting MPTP opening and thereby providing a pharmacological basis for future research and treatment of MIRI.

\section{Acknowledgements}

Not applicable.

\section{Funding}

This study was supported by the National Natural Science Foundation of China (grant no. 81670320 and 81800232) and the Natural Science Foundation of Liaoning Province (grant no. 201602826).

\section{Availability of data and materials}

All datasets used and/or analyzed during the current study are available from the corresponding author on reasonable request.

\section{Authors' contributions}

DJ and NW designed the experiments. JM, SL, ZH and XL performed the experiments. JM, PJ and YG analyzed the data. JM prepared the manuscript. NW revised the manuscript. All authors read and approved the manuscript and agree to be accountable for all aspects of the research in ensuring that the accuracy or integrity of any part of the work are appropriately investigated and resolved.

\section{Ethics approval and consent to participate}

All rats were treated in accordance with the Guide for the Care and Use of Laboratory Animals. The experimental protocol was approved by the Institutional Ethics Committee of China Medical University (Shenyang, China).

\section{Patient consent for publication}

Not applicable.

\section{Competing interests}

The authors declare that they have no competing interests.

\section{References}

1. Moran AE, Forouzanfar MH, Roth GA, Mensah GA, Ezzati M, Murray CJ and Naghavi M: Temporal trends in ischemic heart disease mortality in 21 world regions, 1980 to 2010: The Global Burden of Disease 2010 study. Circulation 129: 1483-1492, 2014.

2. Bainey KR and Armstrong PW: Clinical perspectives on reperfusion injury in acute myocardial infarction. Am Heart J 167: 637-645, 2014.

3. Xu Y, Wu L, Chen A, Xu C and Feng Q: Protective effects of olive leaf extract on acrolein-exacerbated myocardial infarction via an endoplasmic reticulum stress pathway. Int J Mol Sci 19: pii: E493, 2018.

4. Bulluck H, Yellon DM and Hausenloy DJ: Reducing myocardial infarct size: Challenges and future opportunities. Heart 102: 341-348, 2016.

5. Deng X, Xing X, Sun G, Xu X, Wu H, Li G and Sun X: Guanxin danshen formulation protects against myocardial ischemia reperfusion injury-induced left ventricular remodeling by upregulating estrogen receptor $\beta$. Front Pharmacol 8: 777, 2017.

6. Li Y, Xiang Y, Zhang S, Wang Y, Yang J, Liu W and Xue F: Intramyocardial injection of thioredoxin 2-expressing lentivirus alleviates myocardial ischemia-reperfusion injury in rats. Am J Transl Res 9: 4428-4439, 2017.

7. Liu H, Cala PM and Anderson SE: Na/H exchange inhibition protects newborn heart from ischemia/reperfusion injury by limiting $\mathrm{Na}^{+}$-dependent $\mathrm{Ca}^{2+}$ overload. J Cardiovase Pharmacol 55: 227-233, 2010.

8. Cadenas S: ROS and redox signaling in myocardial ischemia-reperfusion injury and cardioprotection. Free Radic Biol Med 117: 76-89, 2018.

9. Griffiths EJ and Halestrap AP: Mitochondrial non-specific pores remain closed during cardiac ischaemia, but open upon reperfusion. Biochem J 307: 93-98, 1995.

10. Javadov S, Jang S, Parodi-Rullán R, Khuchua Z and Kuznetsov AV: Mitochondrial permeability transition in cardiac ischemia-reperfusion: Whether cyclophilin $\mathrm{D}$ is a viable target for cardioprotection? Cell Mol Life Sci 74: 2795-2813, 2017. 
11. Duan X, Ji B, Yu K, Liu J, Hei F and Long C: Pharmacological postconditioning protects isolated rat hearts against ischemia-reperfusion injury: The role of mitochondrial permeability transition pore. ASAIO J 57: 197-202, 2011.

12. Li X, Chen Z, Wu Y, Yan Y, Sun X and Yuan Q: Establishing an artificial pathway for efficient biosynthesis of hydroxytyrosol. ACS Synth Biol 7: 647-654, 2018.

13. Poudyal H, Lemonakis N, Efentakis P, Gikas E, Halabalaki M, Andreadou I, Skaltsounis L and Brown L: Hydroxytyrosol ameliorates metabolic, cardiovascular and liver changes in a rat model of diet-induced metabolic syndrome: Pharmacological and metabolism-based investigation. Pharmacol Res 117: 32-45, 2017.

14. Sun Y, Zhou D and Shahidi F: Antioxidant properties of tyrosol and hydroxytyrosol saturated fatty acid esters. Food Chem 245 1262-1268, 2018.

15. González-Correa JA, Rodríguez-Pérez MD, Márquez-Estrada L, López-Villodres JA, Reyes JJ, Rodriguez-Gutierrez G, Fernández-Bolaños J and De La Cruz JP: Neuroprotective effect of hydroxytyrosol in experimental diabetic retinopathy: Relationship with cardiovascular biomarkers. J Agric Food Chem 66: 637-644, 2018.

16. Zhi LQ, Yao SX, Liu HL, Li M, Duan N and Ma JB: Hydroxytyrosol inhibits the inflammatory response of osteoarthritis chondrocytes via SIRT6-mediated autophagy. Mol Med Rep 17: 4035-4042, 2018.

17. Zubair H, Bhardwaj A, Ahmad A, Srivastava SK, Khan MA, Patel GK, Singh S and Singh AP: Hydroxytyrosol induces apoptosis and cell cycle arrest and suppresses multiple oncogenic signaling pathways in prostate cancer cells. Nutr Cancer 69: 932-942, 2017.

18. Pan S, Liu L, Pan H, Ma Y, Wang D, Kang K, Wang J, Sun B, Sun $\mathrm{X}$ and Jiang H: Protective effects of hydroxytyrosol on liver ischemia/reperfusion injury in mice. Mol Nutr Food Res 57: 1218-1227, 2013

19. Soto-Alarcon SA, Valenzuela R, Valenzuela A and Videla LA: Liver protective effects of extra virgin olive oil: Interaction between its chemical composition and the cell-signaling pathways involved in protection. Endocr Metab Immune Disord Drug Targets 18: 75-84, 2018.

20. Pei YH, Chen J, Xie L, Cai XM, Yang RH, Wang X and Gong JB Hydroxytyrosol protects against myocardial ischemia/reperfusion injury through a PI3K/Akt-dependent mechanism. Mediators Inflamm 2016: 1232103, 2016.

21. Kastenmayer RJ, Moore RM, Bright AL, Torres-Cruz R and Elkins WR: Select agent and toxin regulations: Beyond the eighth edition of the guide for the care and use of laboratory animals. J Am Assoc Lab Anim Sci 51: 333-338, 2012.

22. Wu N, Zhang X, Guan Y, Shu W, Jia P and Jia D: Hypercholesterolemia abrogates the cardioprotection of ischemic postconditioning in isolated rat heart: Roles of glycogen synthase kinase $-3 \beta$ and the mitochondrial permeability transition pore. Cell Biochem Biophys 69: 123-130, 2014.

23. Wu N, Li W, Shu W and Jia D: Protective effect of picroside II on myocardial ischemia reperfusion injury in rats. Drug Des Devel Ther 8: 545-554, 2014.

24. Endlicher R, Kriváková P, Lotkova H, Milerová M, Drahota Z and Cervinková Z: Tissue specific sensitivity of mitochondrial permeability transition pore to $\mathrm{Ca}^{2+}$ ions. Acta Medica (Hradec Kralove) 52: 69-72, 2009.

25. He F, Wu Q, Xu B, Wang X, Wu J, Huang L and Cheng J: Suppression of Stim1 reduced intracellular calcium concentration and attenuated hypoxia/reoxygenation induced apoptosis in H9C2 cells. Biosci Rep 37: pii: BSR20171249, 2017.

26. Hurst S, Hoek J and Sheu SS: Mitochondrial $\mathrm{Ca}^{2+}$ and regulation of the permeability transition pore. J Bioenerg Biomembr 49: 27-47, 2017.

27. Petit PX, Susin SA, Zamzami N, Mignotte B and Kroemer G: Mitochondria and programmed cell death: Back to the future. FEBS Lett 396: 7-13, 1996.

28. Tong Z, Xie Y, He M, Ma W, Zhou Y, Lai S, Meng Y and Liao Z: VDAC1 deacetylation is involved in the protective effects of resveratrol against mitochondria-mediated apoptosis in cardiomyocytes subjected to anoxia/reoxygenation injury. Biomed Pharmacother 95: 77-83, 2017.

29. Penna C, Perrelli MG and Pagliaro P: Mitochondrial pathways, permeability transition pore, and redox signaling in cardioprotection: Therapeutic implications. Antioxid Redox Signal 18: 556-599, 2013.
30. Siemen D and Ziemer M: What is the nature of the mitochondrial permeability transition pore and what is it not? IUBMB Life 65 : 255-262, 2013.

31. Kwong JQ and Molkentin JD: Physiological and pathological roles of the mitochondrial permeability transition pore in the heart. Cell Metab 21: 206-214, 2015.

32. Carraro $M$ and Bernardi P: Calcium and reactive oxygen species in regulation of the mitochondrial permeability transition and of programmed cell death in yeast. Cell Calcium 60: 102-107, 2016.

33. Bernardi P, Rasola A, Forte M and Lippe G: The mitochondrial permeability transition pore: Channel formation by F-ATP synthase, integration in signal transduction, and role in pathophysiology. Physiol Rev 95: 1111-1155, 2015.

34. Morciano G, Bonora M, Campo G, Aquila G, Rizzo P, Giorgi C, Wieckowski MR and Pinton P: Mechanistic role of mPTP in ischemia-reperfusion injury. Adv Exp Med Biol 982: 169-189, 2017.

35. Bopassa JC, Michel P, Gateau-Roesch O, Ovize M and Ferrera R: Low-pressure reperfusion alters mitochondrial permeability transition. Am J Physiol Heart Circ Physiol 288: H2750-H2755, 2005.

36. Fang R, Zhang LL, Zhang LZ, Li W, Li M and Wen K: Sphingosine 1-phosphate postconditioning protects against myocardial ischemia/reperfusion injury in rats via mitochondrial signaling and Akt-Gsk3 $\beta$ phosphorylation. Arch Med Res 48: $147-155,2017$

37. Wang H, Zhao YT, Zhang S, Dubielecka PM, Du J, Yano N, Chin YE, Zhuang S, Qin G and Zhao TC: Irisin plays a pivotal role to protect the heart against ischemia and reperfusion injury. J Cell Physiol 232: 3775-3785, 2017.

38. Zhou H, Zhang Y, Hu S, Shi C, Zhu P, Ma Q, Jin Q, Cao F, Tian F and Chen Y: Melatonin protects cardiac microvasculature against ischemia/reperfusion injury via suppression of mitochondrial fiss ion-VDAC1-HK2-mPTP-mitophagy axis. J Pineal Res 63, 2017.

39. Liu P and Dong J: Protective effects of carnosic acid against mitochondria-mediated injury in H9c2 cardiomyocytes induced by hypoxia/reoxygenation. Exp Ther Med 14: 5629-5634, 2017.

40. Marchi S and Pinton P: The mitochondrial calcium uniporter complex: Molecular components, structure and physiopathological implications. J Physiol 592: 829-839, 2014.

41. Soni M, Prakash C, Dabur R and Kumar V: Protective effect of hydroxytyrosol against oxidativestress mediated by arsenic-induced neurotoxicity in rats. Appl Biochem Biotechnol 186: 27-39, 2018.

42. Siddiqui WA, Ahad A and Ahsan H: The mystery of BCL2 family: Bcl-2 proteins and apoptosis: An update. Arch Toxicol 89: 289-317, 2015.

43. Czabotar PE, Lessene G, Strasser A and Adams JM: Control of apoptosis by the BCL-2 protein family: Implications for physiology and therapy. Nat Rev Mol Cell Biol 15: 49-63, 2014.

44. Narita M, Shimizu S, Ito T, Chittenden T, Lutz RJ, Matsuda H and Tsujimoto Y: Bax interacts with the permeability transition pore to induce permeability transition and cytochrome $c$ release in isolated mitochondria. Proc Natl Acad Sci USA 95: 14681-14686, 1998.

45. Ashkenazi A, Fairbrother WJ, Leverson JD and Souers AJ: From basic apoptosis discoveries to advanced selective BCL-2 family inhibitors. Nat Rev Drug Discov 16: 273-284, 2017.

46. Li H, Sun JJ, Chen GY, Wang WW, Xie ZT, Tang GF and Wei SD: Carnosic acid nanoparticles suppress liver ischemia/reperfusion injury by inhibition of ROS, Caspases and NF- $\kappa \mathrm{B}$ signaling pathway in mice. Biomed Pharmacother 82: 237-246, 2016.

47. Pastorino JG, Tafani M, Rothman RJ, Marcinkeviciute A, Hoek JB and Farber JL: Functional consequences of the sustained or transient activation by Bax of the mitochondrial permeability transition pore. J Biol Chem 274: 31734-31739, 1999.

48. Liao P, Sun G, Zhang C, Wang M, Sun Y, Zhou Y, Sun X and Jian J: Bauhinia championii flavone attenuates hypoxia-reoxygenation induced apoptosis in H9c2 cardiomyocytes by improving mitochondrial dysfunction. Molecules 21: pii: E1469, 2016.

This work is licensed under a Creative Commons Attribution-NonCommercial-NoDerivatives 4.0 International (CC BY-NC-ND 4.0) License. 